\title{
Synthesis of a [1,4]dioxane-2,5-dione based-peptidomimetic scaffold
}

\author{
Andrew D. Abell* and Andrew J. Harvey \\ Department of Chemistry, University of Canterbury, Private Bag 4800, Christchurch, New \\ Zealand \\ Email: andrew.abell@,canterbury.ac.nz
}

\section{Dedicated to Jim Coxon on the occasion of his 65th birthday}

\begin{abstract}
(2S,3S)-3-[N-Cbz-L-Leucinyl]amino-2-hydroxy-4-phenylbutanoic acid 5 dimerised on treatment with EDCI and HOBT to give the symmetrical [1,4]dioxane-2,5-dione-based peptidomimetic 6.
\end{abstract}

Keywords: Peptidomimetic scaffold, synthesis, dimer, cyclisation

\section{Introduction}

An endless array of organic compounds exist that exhibit an equally endless array of biological activities. These compounds function by simply presenting a well-defined topology to a complementary receptor or enzyme in spite of a diversity of molecular structure and biological mechanism of action. It therefore follows that new classes of bioactives would result from simply generating new molecular topologies. One way to achieve this objective is to tether known bioactive molecules onto an appropriate molecular scaffold to generate a new, overall molecular topology. The choice of scaffold needs to allow the ready chemical attachment of a range of simple molecules that possess a diversity of structure and function, perhaps in a combinatorial fashion. The result of such a union is a compound whose overall topology is controlled by the nature and combination of its constituents.

This idea has been used to generate important bioactives that contain a core scaffold and a range of attached ligands that can number anywhere from a few (e.g. protease inhibitors that possess a core isosteric unit to which are attached enzyme binding domains) ${ }^{1}$ to many (e.g. a dendrimer). ${ }^{2}$ Such hybrid compounds provide leads for important biological probes and potential therapeutics with improved potency, stability and in many cases, vastly different biological properties and mechanisms of action relative to their separate components. There is, therefore, a clear need for new and general molecular scaffolds. In this paper we present a simple example of this idea that allows a peptide, or conceivably another suitable functionalized molecule, to be 
attached at two sites on a heterocyclic scaffold (see compound 6). The compound presented here is simple to prepare as a crystalline solid, and demonstrates this principle. Closely related compounds have been reported as inhibitors of Calpains, ${ }^{3}$ an over activity of which is associated with a number of important human diseases. ${ }^{4}$

\section{Results and Discussion}

The optically active peptidomimetic scaffold $\mathbf{6}$ was prepared as detailed in the Scheme below. In the key step, a solution of (2S,3S)-3-[N-Cbz-L-leucinyl]amino-2-hydroxy-4-phenylbutanoic acid 5 in DMF was reacted with a solution of 1-ethyl-3-[3-(dimethylamino)propyl]carbodiimide hydrochloride (EDCI) and hydroxybenztriazole (HOBT) in dichloromethane containing diisopropylethylamine (DIEA) to give 6 as a stable colorless solid in 74\% yield. The starting material 5 was prepared in three steps as shown in the Scheme. The known ${ }^{5} \alpha$-hydroxy $\beta$-amino acid $\mathbf{2}$ was $N$-deprotected with $\mathrm{HBr}$ in acetic acid to give a mixture of $\mathbf{3 a}$ and $\mathbf{3 b}$ (9:1), which was subsequently coupled with $N$-Cbz-L-leucine under standard EDCI conditions to give $4 \mathbf{a}$ and $\mathbf{4 b}$ (9:1). This mixture was finally hydrolysed to give the desired derivative 5 (see step iii) in $69 \%$ over two steps. It is interesting to note that the ratio of $\mathbf{3 a}$ to $\mathbf{3 b}$ obtained in step i was dependent upon the concentration of 2 used in the reaction. Product ratios of 4:1, 9:1, 12:1 and 19:1 were obtained for concentrations of $0.26,0.97,1.47$ and $2.00 \mathrm{M}$, respectively.

The dimeric nature of $\mathbf{6}$ was confirmed by a combination of ${ }^{1} \mathrm{H}$ NMR, ${ }^{13} \mathrm{C}$ NMR and mass spectrometry. The two-fold axis of symmetry in the dimer 6 was confirmed by the presence of only twenty peaks in the ${ }^{13} \mathrm{C}$ NMR spectrum while high resolution mass spectral data was consistent with the dimeric structural formula $\left[\mathrm{C}_{48} \mathrm{H}_{57} \mathrm{O}_{10} \mathrm{~N}_{4}(\mathrm{M}+1)^{+}\right]$. In addition, the ${ }^{1} \mathrm{H}$ NMR of 6 revealed a resonance at 5.49 ppm that was shown to correspond with $\mathrm{H} 2$ by a $2 \mathrm{D}$ NMR COSY experiment - a key correlation was observed in the COSY experiment between H3 (4.66 ppm) and H2 (5.49 ppm). Importantly, the chemical shift of H2 is consistent with O-acylation ${ }^{6}$ where a similar chemical shift was observed for $\mathrm{H} 2$ in $\mathbf{4 b}(5.53 \mathrm{ppm})$, whereas $\mathrm{H} 2$ in the corresponding alcohol 4 a resonated further upfield at $4.29 \mathrm{ppm}$.

In this paper we have presented the synthesis of an interesting and new class of peptidomimetic molecular scaffold whose architecture should be amenable to attaching a wide range of amino acid $\mathrm{R}$ groups and thus the presentation of a diverse range of topologies. 

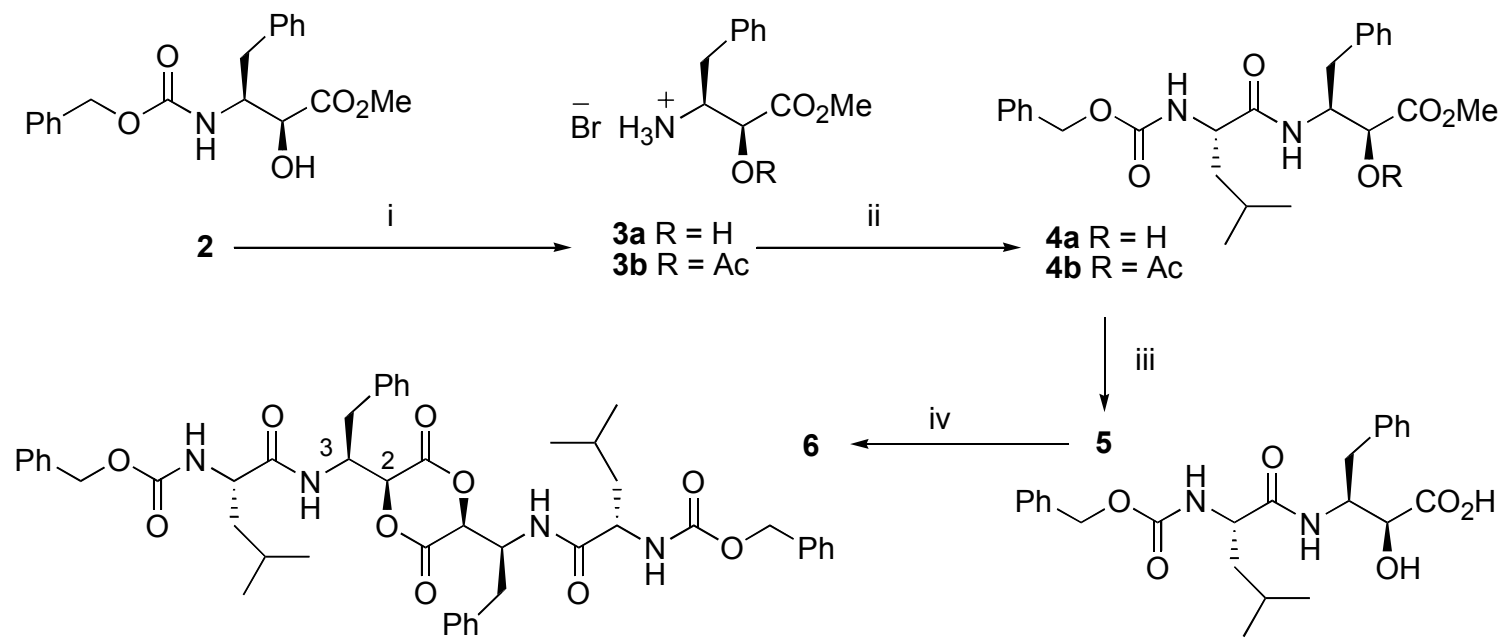

Sheme 1. Reagents and conditions: (i) 33\% HBr in AcOH, 20 min, 9 (3a):1 (3b); (ii) N-Cbz-LLeu, EDCI, HOBT, DIEA, DMF, DCM, 16 h, 9 (4a): 1 (4b); (iii) LiOH, $\mathrm{MeOH}, \mathrm{H}_{2} \mathrm{O}$, 4h; (iv) EDCO, HOBT, DIEA, DMF, DCM $<16$ h, $74 \%$.

\section{Experimental Section}

General Procedures. ${ }^{1} \mathrm{H}$ NMR spectra were obtained on a Varian Inova spectrometer, operating at $500 \mathrm{MHz}$. Coupling constants $(J)$ are quoted in $\mathrm{Hz} .{ }^{13} \mathrm{C}$ NMR spectra were obtained on a Varian Unity 300 spectrometer, operating at $75 \mathrm{MHz}$, with a delay $\left(\mathrm{D}_{1}\right)$ of $1 \mathrm{~s}$. Electron impact (EI) mass spectra were detected on a Kratos MS80 RFA mass spectrometer operating at $4000 \mathrm{~V}$ (accelerating potential) and $70 \mathrm{eV}$ (ionisation energy) using a source temperature of $200-250^{\circ} \mathrm{C}$.

(2S,3S)-3-[N-Cbz-L-Leucinyl]amino-2-hydroxybenzenebutanoic acid (5). Compound $\mathbf{2}^{5}$ (500 $\mathrm{mg}, 1.47 \mathrm{mmol})$ was dissolved in a solution of $\mathrm{HBr}$ in acetic acid $(33 \%, 1.0 \mathrm{ml})$ and the resulting mixture was stirred at $\mathrm{rt}$ for $20 \mathrm{~min}$. The addition of ether $(2 \mathrm{~mL})$ caused the amine salt to precipitate. The solvent was drawn off and the resulting residue retained. To the etheral solution was added petroleum ether $(4 \mathrm{~mL})$ to give a further precipitate. The solvent was drawn off and the two batches of solid were combined and dried to give a mixture (397 mg, 12:1 by ${ }^{1} \mathrm{H} \mathrm{NMR}$ ) of the amine salts $\mathbf{3 a}(86 \%)$ and $\mathbf{3 b}(6 \%)$ as a brown tar. Selected data from the mixture: ${ }^{1} \mathrm{H}$ NMR 3a $\left(\mathrm{D}_{2} \mathrm{O}\right) \delta 7.19-7.31(5 \mathrm{H}, \mathrm{m}, \mathrm{ArH}), 4.51(1 \mathrm{H}, \mathrm{d}, \mathrm{J}=2.5 \mathrm{~Hz}, \mathrm{CHCHOH}), 4.03(1 \mathrm{H}, \mathrm{m}$, $\mathrm{CHCHOH}), 3.38\left(3 \mathrm{H}, \mathrm{s}, \mathrm{CH}_{3}\right), 2.89\left(2 \mathrm{H}, \mathrm{m}, \mathrm{CH}_{2} \mathrm{Ph}\right) .{ }^{1} \mathrm{H}$ NMR $3 \mathbf{b}\left(\mathrm{D}_{2} \mathrm{O}\right) \delta$ 7.19-7.31 (5H, m, ArH), $5.41(1 \mathrm{H}, \mathrm{d}, \mathrm{J}=1.7 \mathrm{~Hz}, \mathrm{CHCHOAc}), 4.25$ (1H, m, CHCHOAc), $3.41\left(3 \mathrm{H}, \mathrm{s}, \mathrm{COOCH}_{3}\right)$, $3.00\left(2 \mathrm{H}, \mathrm{m}, \mathrm{CH}_{2} \mathrm{Ph}\right), 2.18\left(3 \mathrm{H}, \mathrm{s}, \mathrm{OCOCH}_{3}\right)$.

To a mixture of 3a and $\mathbf{3 b}\left(64 \mathrm{mg}, 9: 1\right.$ by ${ }^{1} \mathrm{H}$ NMR, $0.22 \mathrm{mmol}$ ) were added EDCI (47 mg, $0.25 \mathrm{mmol}$ ) and HOBT (32 mg, $0.24 \mathrm{mmol})$. A solution $(1.0 \mathrm{M})$ of $N$-Cbz- $L$-leucine in $N$ methylpyrrolidinone $(240 \mu \mathrm{L}, 0.24 \mathrm{mmol})$, DMF $(250 \mu \mathrm{L})$ and DIEA (43 $\mu \mathrm{L}, 0.25 \mathrm{mmol})$ were then added and the reaction mixture was stirred overnight at $\mathrm{rt}$ under nitrogen. The reaction 
mixture was diluted with ethyl acetate $(4 \mathrm{~mL})$ and washed with $10 \%$ aqueous $\mathrm{HCl}(3 \times 2 \mathrm{~mL})$, saturated $\mathrm{NaHCO}_{3}(3 \times 2 \mathrm{~mL})$, brine $(2 \mathrm{~mL})$, dried over $\mathrm{MgSO}_{4}$ and concentrated in vacuo to give a mixture (9:1 by ${ }^{1} \mathrm{H}$ NMR) of $\mathbf{4 a}$ and $\mathbf{4 b}(69 \mathrm{mg}, 69 \%)$ as a white solid. Data from the mixture: [MS (FAB): $499\left[(\mathrm{M}+1)^{+}\right.$4b], $457\left[(\mathrm{M}+1)^{+}\right.$4a] $\left., 414,307,210,154,136\right]$; mp 138-144 ${ }^{\circ} \mathrm{C}$; IR $\left(\mathrm{CHCl}_{3}\right) 3688,3416,2961,1734,1676,1601,1500,1440 \mathrm{~cm}^{-1} .{ }^{1} \mathrm{H}$ NMR 4a $\left(\mathrm{CDCl}_{3}\right) \delta$ 7.14-7.35 (10H, m, ArH), $6.51(1 \mathrm{H}, \mathrm{d}, \mathrm{J}=8.8 \mathrm{~Hz}, \mathrm{PheNH}), 5.09$ (3H, m, CbzCH 2 \& CbzNH), $4.51(1 \mathrm{H}, \mathrm{ddd}, \mathrm{J}=2.9,7.3,16.1 \mathrm{~Hz}, \mathrm{CHCHOH}), 4.29(1 \mathrm{H}$, br s, CHCHOH$), 4.11(1 \mathrm{H}, \mathrm{m}$, NHCHCO), 3.59 (3H, s, $\left.\mathrm{OCH}_{3}\right), 2.79\left(2 \mathrm{H}, \mathrm{m}, \mathrm{PheCH}_{2}\right), 1.61\left(3 \mathrm{H}, \mathrm{m}, \mathrm{LeuCH} \& \mathrm{LeuCH}_{\mathrm{A}}\right), 1.39$ $\left(1 \mathrm{H}, \mathrm{m}, \mathrm{LeuCH}{ }_{\mathrm{B}}\right), 0.89(6 \mathrm{H}, \mathrm{m}, 2 \mathrm{x} \mathrm{LeuCH}) ;{ }^{13} \mathrm{C} \mathrm{NMR} 4 \mathbf{a}\left(\mathrm{CDCl}_{3}\right) \delta 172.8(\mathrm{COOMe}), 172.0$ (NHCHCO), 156.2 (CbzCO), 136.7 (PheArC), 136.0 (CbzArC), 129.3, 128.5, 128.3, 128.3, 128.1, 126.7 (ArCH), $72.0(\mathrm{CHOH}), 67.2\left(\mathrm{CbzCH}_{2}\right), 53.7(\mathrm{NHCHCO}), 53.0(\mathrm{CHCHOH}), 52.5$ $\left(\mathrm{COOCH}_{3}\right), 41.0\left(\mathrm{LeuCH}_{2}\right), 35.4\left(\mathrm{PheCH}_{2}\right), 24.6(\mathrm{LeuCH}), 22.8\left(\mathrm{Leu}\left(\mathrm{CH}_{3}\right)_{\mathrm{A}}\right), 21.9\left(\mathrm{Leu}\left(\mathrm{CH}_{3}\right)_{\mathrm{B}}\right)$. ${ }^{1} \mathrm{H}$ NMR 4b $\left(\mathrm{CDCl}_{3}\right) \delta 6.42(1 \mathrm{H}, \mathrm{m}, \mathrm{PheNH}), 5.53(1 \mathrm{H}, \mathrm{s}, \mathrm{CHOAc}), 3.63\left(3 \mathrm{H}, \mathrm{s}, \mathrm{COOCH}_{3}\right), 2.16$ $\left(3 \mathrm{H}, \mathrm{s}, \mathrm{OCOCH}_{3}\right)$.

To the mixture of $\mathbf{4 a}$ and $\mathbf{4 b}\left(60 \mathrm{mg}, 0.13 \mathrm{mmol}, 9: 1\right.$ by $\left.{ }^{1} \mathrm{H} \mathrm{NMR}\right)$ at $0{ }^{\circ} \mathrm{C}$ was added a solution of $0.25 \mathrm{M}$ lithium hydroxide (1.5 parts) in 2:1 methanol/water. After the reaction mixture was stirred for $0.5-4 \mathrm{~h}$, sufficient $1 \mathrm{~N}$ aqueous $\mathrm{HCl}$ was added to acidify the solution $(\mathrm{pH}$ 5 by Universal Indicator) and the methanol was evaporated in vacuo. Water was added to the residue and the organics were extracted with ethyl acetate. The combined organic extracts were washed with water, brine, dried over $\mathrm{MgSO}_{4}$ and concentrated in vacuo to the acid 5 (58 $\mathrm{mg}$, qu) as a white solid. ${ }^{1} \mathrm{H}$ NMR $\left(\mathrm{CD}_{3} \mathrm{OD}\right) \delta 7.82(1 \mathrm{H}, \mathrm{d}, \mathrm{J}=9.3 \mathrm{~Hz}, \mathrm{CbzNH}), 7.33(5 \mathrm{H}, \mathrm{m}, \mathrm{ArH}), 7.10$ $7.19(5 \mathrm{H}, \mathrm{m}, \mathrm{ArH}), 5.07\left(2 \mathrm{H}, \mathrm{m}, \mathrm{CbzCH}_{2}\right), 4.49(1 \mathrm{H}, \mathrm{m}, \mathrm{CHCHOH}), 4.17(1 \mathrm{H}, \mathrm{d}, \mathrm{J}=4.4 \mathrm{~Hz}$, $\mathrm{CHCHOH}), 4.07$ (1H, m, NHCHCO), $2.80\left(2 \mathrm{H}, \mathrm{m}, \mathrm{PheCH}_{2}\right), 1.58(1 \mathrm{H}, \mathrm{m}, \mathrm{LeuCH}), 1.33(2 \mathrm{H}$,

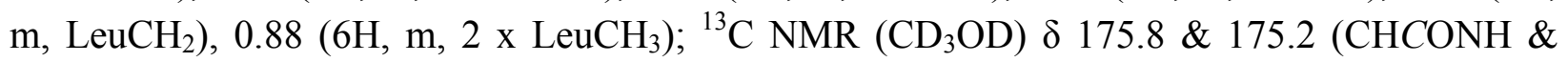
$\mathrm{COOH}$ ), 158.6 (CbzCO), 139.6 (PheArC), 138.4 (CbzArC), 130.7, 129.8, 129.6, 129.3, 129.2, $127.6(\mathrm{ArCH}), 73.9(\mathrm{CHOH}), 68.0\left(\mathrm{CbzCH}_{2}\right), 55.4(\mathrm{NHCHCO}), 54.8(\mathrm{CHCHOH}), 42.5$ $\left(\mathrm{LeuCH}_{2}\right), 36.4\left(\mathrm{PheCH}_{2}\right), 26.1(\mathrm{LeuCH}), 23.6\left(\mathrm{Leu}\left(\mathrm{CH}_{3}\right)_{\mathrm{A}}\right), 22.2\left(\mathrm{Leu}\left(\mathrm{CH}_{3}\right)_{\mathrm{B}}\right)$; [MS $(\mathrm{FAB}): 465$ $(\mathrm{M}+\mathrm{Na})^{+}, 443,399,286,176,153,136$; HRMS (FAB) calcd for $\mathrm{C}_{24} \mathrm{H}_{30} \mathrm{O}_{6} \mathrm{~N}_{2} \mathrm{Na}(\mathrm{M}+\mathrm{Na})^{+}$ 465.2002, found 465.1996]; mp 131-134 ${ }^{\circ} \mathrm{C}$; IR $\left(\mathrm{CHCl}_{3}\right)$ 3433, 2963, 1720, 1660, 1510, 1456, $1340,1217 \mathrm{~cm}^{-1}$.

Dimer 6. To a solution of 5 (14 mg, $32 \mu \mathrm{mol})$ in DMF $(1 \mathrm{~mL})$ was added EDCI $(8 \mathrm{mg}, 41 \mu \mathrm{mol})$, HOBT $(7 \mathrm{mg}, 47 \mu \mathrm{mol})$, dichloromethane $(1 \mathrm{~mL})$ and DIEA $(6 \mu \mathrm{L}, 35 \mu \mathrm{mol})$. The reaction mixture was stirred overnight at $\mathrm{rt}$ before being diluted with dichloromethane $(10 \mathrm{~mL})$, washed with $10 \%$ aqueous $\mathrm{HCl}(10 \mathrm{~mL})$, brine $(10 \mathrm{~mL})$, dried over $\mathrm{MgSO}_{4}$, and concentrated in vacuo to afford 6 (10 mg, 74\%) as a white solid. ${ }^{1} \mathrm{H}$ NMR (300Mz, DMSO) $\delta 8.28(2 \mathrm{H}, \mathrm{d}, \mathrm{J}=8.2 \mathrm{~Hz}$, PheNH), 7.16-7.35 (22H, m, arom \& CbzNH), 5.49 (2H, brs, H2), $5.01\left(4 \mathrm{H}, \mathrm{s}, \mathrm{CbzCH}_{2}\right), 4.66$ $(2 \mathrm{H}, \mathrm{m}, \mathrm{H} 3), 4.05(2 \mathrm{H}, \mathrm{m}, \mathrm{Leu}-\alpha \mathrm{H}), 2.81\left(4 \mathrm{H}, \mathrm{m}, \mathrm{PheCH}_{2}\right), 1.49$ (2H, m, LeuCH), 1.36 - 1.27 $\left(4 \mathrm{H}, \mathrm{m}, \mathrm{LeuCH}_{2}\right), 0.81\left(12 \mathrm{H}, \mathrm{m}, \mathrm{LeuCH}_{3}\right) ;{ }^{13} \mathrm{C} \mathrm{NMR}$ (DMSO) $\delta 172.1$ (ring CO), 165.7 (LeuCO), 155.4 (CbzCO), 137.6, 136.7, 128.7, 128.1, 128.0, 127.6, 127.5, 126.1, 77.6 (C2), 65.2 $\left(\mathrm{CbzCH}_{2}\right), 52.8$ (Leu- $\left.\alpha \mathrm{C}\right), 49.9(\mathrm{C} 3), 40.7\left(\mathrm{LeuCH}_{2}\right), 33.8\left(\mathrm{PheCH}_{2}\right), 23.9(\mathrm{LeuCH}), 22.6$ 
$\left(\mathrm{LeuCH}_{3}\right), 21.3\left(\mathrm{LeuCH}_{3}\right)$; [MS (ES): $871(\mathrm{M}+\mathrm{Na})^{+}, 849(\mathrm{M}+1)^{+}, 647,556,537,464,316,289$, 275, 249; HRMS (ES) calcd for $\mathrm{C}_{48} \mathrm{H}_{57} \mathrm{O}_{10} \mathrm{~N}_{4}(\mathrm{M}+1)^{+}$849.408, found 849.405]; $\mathrm{R}_{\mathrm{F}} 0.19$ (1:4 ethyl acetate-dichloromethane); IR $\left(\mathrm{CHCl}_{3}\right) 3628,3354,1767,1722,1683,1228 \mathrm{~cm}^{-1} ;[\alpha]_{\mathrm{D}}-78^{\circ}$ (c 0.082 acetonitrile).

\section{Acknowledgements}

The financial support of a Royal Society of New Zealand Marsden grant is gratefully acknowledged.

\section{References}

1. (a)Abdel-Rahman, H. M.; Al-karamany, G. S.; El-Koussi, N. A.; Youssef, A. F.; Kiso, Y.; Curr. Med. Chem. 2002, 9, 1905. (b)Lam, P. Y. S.; Jadhav, P. K.; Eyermann, C. J.; Hodge, C, N.; Ru, Y.; Bacheler, L. T.; Meek, J. L.; Otto, M. J.; Rayner, M. M.; Wong, Y. N.; Chang, C. H.; Weber, P. C.; Jackson, D. A.; Sharpe, T. R.; Ericksonviitanen, S. Science 1994, 263, 380 .

2. (a)Boas, U.; Heegaard, P. M. H. Chem. Soc. Rev. 2004, 33, 43. (b) Dykes, G. M.; J. Chem. Technol. Biotechnol. 2001, 76, 903.

3. Alvarez, M. E.; Houck, D. R.; White, C. B.; Brownell, J. E.; Bobko, M. A.; Rodger, C. A.; Stawicki, M. B.; Sun, H. H.; Gillum, A. M.; Cooper, R. J. Antibiot. 1994, 47, 1195.

4. (a)Lin, G. D.; Chattopadhyay, D.; Maki, M.; Wang, K. K. W.; Carson, M.; Jin, L.; Yuen, P. W.; Takano, E.; Hatanaka, M.; DeLucas, L. J.; Narayana, S. V. L. Nat. Struct. Biol. 1997, 4, 539. (b) Chatterjee, S.; Gu, Z.-Q.; Dunn, D.; Tao, M.; Josef, K.; Tripathy, R.; Bihovsky, R.; Senadhi, S. E.; O'Kane, T. M.; McKenna, B. A.; Mallya, S.; Ator, M. A.; Bozyczko-Coyne, D.; Siman, R.; Mallamo, J. P. J. Med. Chem. 1998, 41, 2663. (c) Mathur, P.; Gupta, S. K.; Wegener, A. R.; Breipohl, W.; Ahrend, M. H.; Sharma, Y. D.; Gupta, Y. K.; Vajpayee, R. B. Curr. Eye Res. 2000, 21, 926.

5. Wasserman, H. H.; Ho, W.-B. J. Org. Chem. 1994, 59, 4364.

6. Kemp, W. Organic Spectroscopy; MacMillan: London, 1991; $3^{\text {rd }}$ Edn., p. 171. 